\title{
La informalidad en la tenencia de la tierra y la producción de vivienda urbana en Facatativá (Cundinamarca) 1995 2009
}

Informality in the land tenure and urban housing production in Facatativá (Cundinamarca) 1995-2009

Ruth Marina Solano Diaz ${ }^{1}$

\section{Resumen}

La apropiación informal del espacio urbano para construir vivienda es un fenómeno que se genera como respuesta a la insatisfacción del derecho fundamental a la vivienda. Dicho suceso se sustenta como producto de unas lógicas de acción social, que se alimentan de tres factores: la pobreza, el precio de la tierra urbana y los programas de formalización de la tenencia. El presente estudio pudo comprobar la hipótesis según la cual los citados factores -que para efectos de este trabajo fueron analizados durante el periodo 1995-2009- han propiciado la informalidad en el sector urbano del municipio de Facatativá, Cundinamarca. Igualmente, se explica por qué aun después de implementar los programas de formalización y los planes de ordenamiento territorial, el fenómeno persiste. Por último, con fundamento en los resultados obtenidos, se proponen alternativas que, se espera, pueden contribuir a prevenir este comportamiento.

Palabras clave: Acción social, formalización, informalidad, pobreza, precio de la tierra, vivienda.

1 Magister en Geografía. Profesional Especializado, Subdirección de Catastro Instituto Geográfico Agustín Codazzi IGAC. Bogotá D.C. - Colombia.rsolano@igac.gov.co. 


\section{Abstract}

The informal ownership of urban space to produce housing is a phenomenon which is generated in response to the dissatisfaction of the fundamental right to housing. This event is based on a logical product of social action, which feeds on three factors: poverty, urban land prices and programs of tenure formalization. This study was able to verify the hypothesis according to which these factors -that for the purposes of this work were analyzed during the period 1995-2009- have contributed to the informality on the urban sector in the municipality of Facatativá, Cundinamarca. This also explains why, even after implementing programs of formalization and land use plans, the phenomenon persists. Finally, based on the results, we propose alternatives that are considered to contribute to prevent this behavior.

Key words: Formalizing, housing, informality, land prices, poverty, social action. 


\section{Introducción}

El desarrollo urbanístico de la ciudad de Facatativá se ha dado bajo procesos constructivos, tanto planificados, como no planificados, siendo estos últimos el resultado de prácticas informales de tenencia de la tierra, que prevalecen aún después de expedidas las normas de ordenamiento territorial pertinentes y que consiguen urbanizar el $19 \%$ del total de los barrios que conforman la ciudad.

Esta manera informal de construir la ciudad se enmarca dentro de tres lógicas de acción social, que Pedro Abramo (2003) define como: la lógica del Estado, con la cual los individuos acatan las decisiones del Estado por considerarlo garante del mayor grado de bienestar social, en el proceso de acceder y usufructuar el suelo urbano; la lógica del mercado, con la cual el mercado es el mecanismo social que posibilita el encuentro entre los que desean "consumir" suelo urbano y los que están dispuestos a ofrecerlo, y la "lógica de la necesidad", con la cual se genera una motivación condicionada por la pobreza, es decir, por la incapacidad de suplir una necesidad básica, de acceso al mercado de vivienda, con los recursos monetarios disponibles.

Al respecto, Duhau (2002) argumenta que con la informalidad coexisten tres componentes principales, derivados de las relaciones políticas, económicas y sociales, que son: las formas de acceso al suelo (por ocupación de hecho), la producción de vivienda (por autoproducción progresiva) y la vida urbana, los cuales determinan contextos de tenencia de la tierra, en los que el Estado busca legitimar la posesión para permitir el acceso a los bienes colectivos urbanos y no para resolver la necesidad de producir vivienda.

El fenómeno de la informalidad es contemplado en la legislación colombiana como una situación anómala que debe ser corregida, pues se considera contraria a los preceptos constitucionales que promueven el acceso progresivo a la propiedad de la tierra y a la garantía de la propiedad privada. En este sentido se observa de una parte que, según directrices del Decreto 755 de 1995, creador del Programa Presidencial para la Formalización de la Propiedad y Modernización de la Titulación Predial, los programas de formalización buscan garantizar la seguridad de la tenencia y fomentar la cohesión social que genera la vivienda, y de otra que los planes de Ordenamiento Territorial, como instrumento regulador del uso equitativo y racional del suelo, se encaminan a alcanzar la función social de la propiedad y el derecho constitucional a la vivienda (Decreto 755, Presidencia de la República, 1995).

Con fundamento en lo citado se analizó el problema de investigación en el periodo 1995-2009, por coincidir la primera fecha con la promulgación del citado decreto, y la segunda, con la ejecución durante siete años del Plan de Ordenamiento Territorial de Facatativá, aprobado en el año 2002. Hechos que permitieron evaluar el alcance y los efectos de la implementación de los programas de regularización y el impacto de 
los mismos frente a los temas de pobreza y precio de la tierra.

En concordancia con lo citado surgen las siguientes preguntas:

- ¿La pobreza propicia la informalidad en la tenencia de la tierra urbana para producir vivienda?

- ¿Los precios de la tierra urbana predisponen la informalidad en la tenencia de la tierra para producir vivienda?

- ¿La aplicación de programas de regularización de la vivienda informal en Facatativá ha contribuido a la permanencia del fenómeno?

\section{La informalidad en la producción de la vivienda}

La informalidad en la construcción del espacio urbano es el resultado de un proceso social en el que participan actores que, por diversas circunstancias, principalmente por la necesidad de disponer de un lugar para instalarse, "lógica de necesidad" (Abramo, 2003), han sido conducidos a adelantar y materializar acciones urbanísticas en contravención de las directrices del ordenamiento territorial. La comprensión de este fenómeno requiere de un acercamiento a las consideradas raíces de la problemática, a fin de propiciar aportes que contribuyan a la generación de políticas que verdaderamente lo controlen y propendan por el bienestar social y el desarrollo económico equitativo de los municipios $\mathrm{y}$ de sus habitantes.

Lo anterior se expresa por cuanto "en la práctica, muchos de los programas de regularización se han desarrollado sin considerar las causas de la informalidad, generando resultados contraproducentes que tienden a acentuar los procesos de segregación socio-espacial" (Fernandes \& Smolka, 2004).

\section{Descripción del lugar}

La investigación se adelantó en la zona urbana de Facatativá, departamento de Cundinamarca, Colombia. Dicha zona cuenta con una extensión de $5 \mathrm{Km}^{2}$, que representa el $3 \%$ de la superficie municipal, la cual albergaba para el año 2005 una población de 95.640 habitantes. Facatativá se localiza entre las coordenadas geográficas $4^{\circ} 48^{\prime} 46^{\prime}$ ' latitud norte y $74^{\circ} 21^{\prime} 00^{\prime}$ ' longitud oeste y en altitudes que oscilan entre $2.600 \mathrm{msnm}$ en su parte plana y $3.200 \mathrm{msnm}$ en sus cerros circundantes, su temperatura promedio es de $14^{\circ} \mathrm{C}$ (IGAC, 1996).

\section{Metodología}

Esta investigación es de tipo documental. Hay que aclarar que en ella está definido como unidad de estudio el sector urbano del municipio de Facatativá, como población, los barrios del sector urbano, y como muestra objeto de investigación, los barrios de origen informal. Entretanto, las variables seleccionadas para explicar el problema fueron los precios de la tierra urbana, la pobreza y los programas 
Figura 1. Organigrama de Procesos

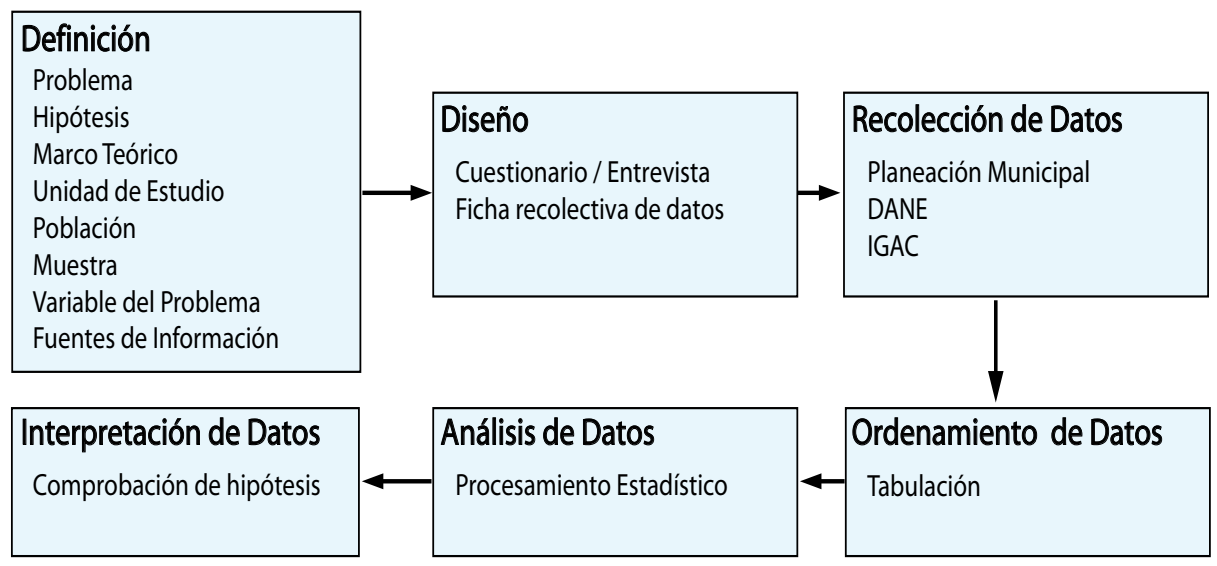

Fuente: Elaboración propia

de formalización; a su vez, las fuentes de dicha información fueron el Instituto Geográfico Agustín Codazzi IGAC, el Departamento Administrativo Nacional de Estadística DANE y la Oficina de Planeación del municipio de Facatativá, respectivamente (ver Figura 1).

La información pertinente a la variable barrios informales se obtuvo del Plan de Ordenamiento Territorial (POT) del municipio de Facatativá, y se complementó mediante encuesta aplicada en la Oficina de Planeación Municipal. Los datos de los programas de formalización se consiguieron por medio de encuesta aplicada en la misma oficina, las cifras de pobreza se tomaron de los reportes del DANE y se relacionaron en un formulario, los valores pertinentes al precio de la tierra se obtuvieron de los estudios hechos por el IGAC acerca de zonas homogéneas físicas y geoeconómicas ZHG del municipio, $\mathrm{y}$ se recopilaron en un formulario.
Los estudios de Zonas Homogéneas Físicas ZHF y Zonas Homogéneas Geoeconómicas ZHG, que desarrolla periódicamente el IGAC, para establecer el valor del terreno y de las construcciones de los inmuebles a nivel municipal, permitieron observar comparativamente la tendencia en los precios de la tierra en Facatativá. Estos estudios se inician con la definición de unas zonas homogéneas físicas $\mathrm{ZHF}$, las cuales hacen referencia a espacios geográficos que agrupan manzanas con similares características en las variables servicios públicos, topografía, vías, uso (residencial, comercial, industrial, etc.) y tipificación, y que son el insumo base para adelantar sobre cada una de ellas la investigación del mercado inmobiliario, a fin de determinar los diferentes valores del terreno (IGAC, 1985).

La mencionada tipificación consiste en una clasificación de las construcciones, que se efectúa a partir de una califica- 
ción tipo, de 1 a 100 puntos, que hace referencia a los materiales constructivos de las viviendas (IGAC, 1997), los cuales están asociados a su diseño. En esta escala de calificación se establecen seis categorías o tipos [1) bajo-bajo (0-10), 2) bajo (11-28), 3) medio-bajo (29-46), 4) medio (47-64), 5) medio alto (6582), y 6) alto (83-100)], que indican, para los menores puntajes, presencia de materiales y diseños más económicos y para los más altos puntajes, diseños y materiales más costosos, permitiendo abstraer las condiciones generales de las edificaciones.

Con las Zonas Homogéneas Físicas ZHF obtenidas y con fundamento en un estudio del mercado inmobiliario del sector, se diseñan unas zonas homogéneas geoeconómicas ZHG, sobre las ZHF, las cuales permiten obtener espacios geográficos que involucran las manzanas que, además de tener características físicas similares, presentan valores por $\mathrm{m}^{2}$ de terreno semejantes, (ver Figura 2).

Figura 2. Mapa de Zonas Homogéneas Geoeconómicas



Fuente: elaboración propia a partir de investigación IGAC 
De otra parte, se establece que las cifras de pobreza reportadas por el DANE son el resultado de aplicar el método de Necesidad Básicas Insatisfechas NBI, el cual busca determinar si las necesidades básicas de la población se encuentran cubiertas, con fundamento en las condiciones de su vivienda; por lo tanto, se deduce que las personas que habitan viviendas con NBI, se encuentran en las mismas condiciones de la vivienda.

Ahora bien, la etapa de análisis de variables se llevó a cabo de la siguiente manera: el estudio de la variable barrio informal se consiguió observando, en cartografía multitemporal, el comportamiento espacial de estos barrios; por su parte, el establecimiento del patrón de ocupación y el examen de la tendencia de los precios de la tierra urbana formal frente a la informal, se hicieron comparando los precios reportados por el IGAC en zonas de origen formal e informal, con características físicas similares, durante el periodo de estudio. Y la eficacia de los programas de formalización se estudió a partir del contexto normativo que fue desarrollado para promover el acceso progresivo a la propiedad de la tierra y para garantizar la propiedad privada, a fin de establecer si dichos programas lograron detener el avance del fenómeno. Por último, la trayectoria de la variable pobreza fue interpretada mediante la combinación de cifras de población y teniendo en cuenta el contexto económico nacional y municipal de la época, para definir si la misma aumentó o disminuyó durante el periodo de estudio.

\section{Resultados}

En consecuencia, con la espacialización proveniente de identificar cartográficamente la ubicación de los barrios informales, se encontró que estos asentamientos se desarrollaron en algunos terrenos ubicados en la periferia de la ciudad, (ver Figura 3). Dicho resultado asociado a la reglamentación urbanística del suelo para la época, permite establecer que estos terrenos hacían parte de la zona rural del municipio y, por ende, su regulación no permitía desarrollos de tipo urbano; no obstante fueron parcelados y vendidos con la expectativa de una supuesta licencia de urbanismo en trámite.

A su vez la indagación pertinente a la variable precios de la tierra, permitió establecer que la dotación de servicios básicos domiciliarios es un componente fundamental para determinar el precio de la tierra urbana y que el mismo genera, para el presente caso, diferencias de nueve veces entre terrenos con tenencia de origen informal (ZHG 15 frente a ZHG 9) y de dos veces entre las zonas de origen informal mejor dotadas y las zonas de origen formal con dotación completa (ZHG 9 frente a ZHG 5), ver Figura 4.

De otra parte, la averiguación sobre los programas de formalización reveló que los primeros procesos de formalización se presentaron en el año 1993, después de 28 años de su conformación, en el marco de la formulación de la política nacional de asentamientos humanos y expansión urbana; como también que dentro del periodo de estudio estaba en proceso la formalización de sectores de cinco barrios 
Figura 3. Asentamientos informales

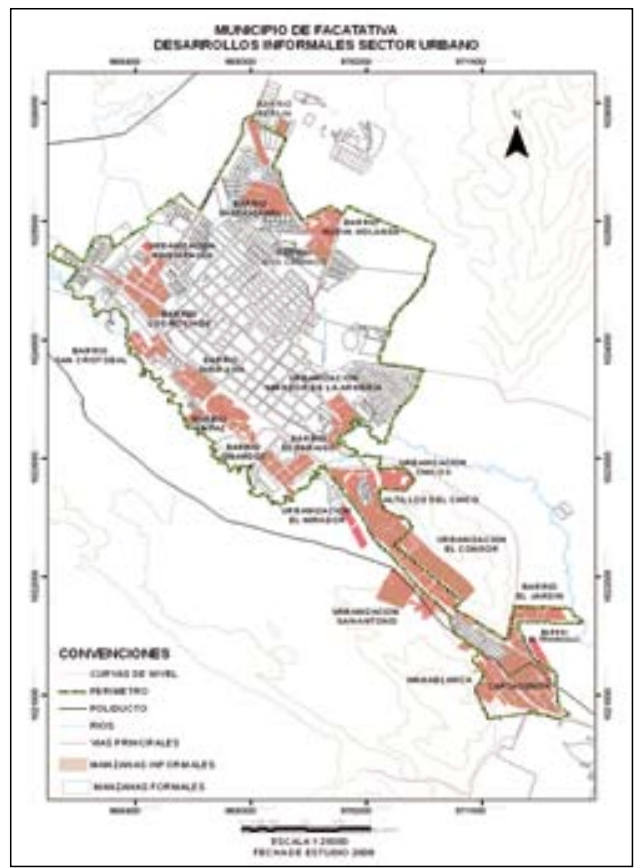

Fuente: elaboración propia a partir de información Oficina Planeación Municipal Facatativá

Figura 4. Evolución precio por $\mathrm{m}^{2}$ terrenos bajo tenencia de origen formal destinados a vivienda ZHG 5, frente a terrenos bajo tenencia de origen informal ZHG 9 y 15.

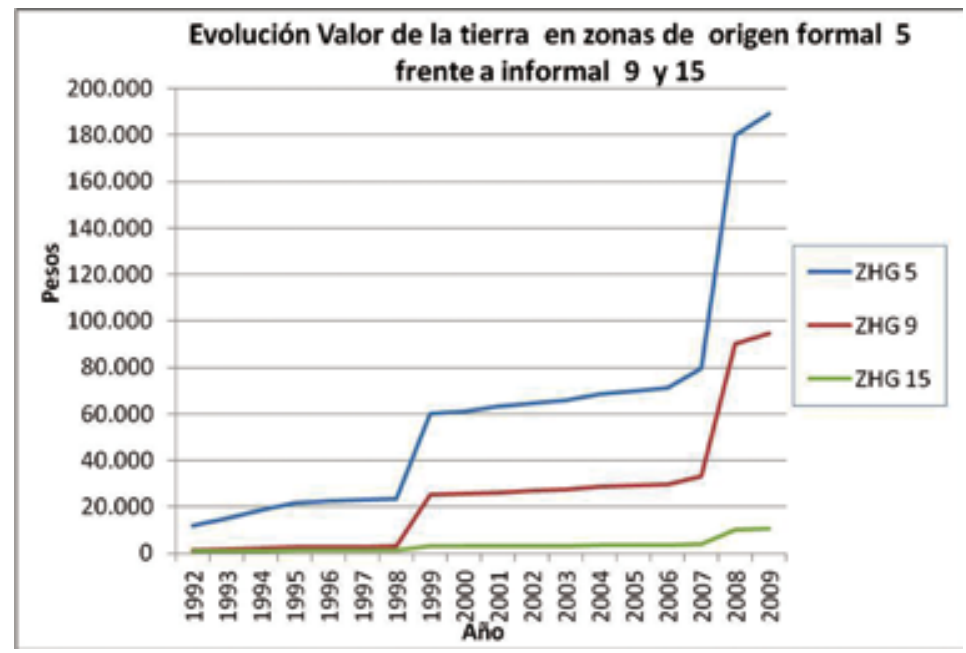

Fuente: elaboración a partir de información IGAC 
erigidos en los años 1979 (1), 1988 (1) y 2000 (3), respectivamente, (ver Tabla 1). Así mismo, se logró observar que los barrios que han alcanzado mayor área ocupada son Cartagenita, Los MolinosMaría Paula y Santa Isabel, los cuales se identifican como pioneros en esta clase de desarrollos urbanísticos.

En desarrollo de la investigación se pudo comprobar que el proceso de subdivisión de fincas aledañas al sector urbano continúa, a pesar de que el artículo 266 del POT de Facatativá, prohíbe los loteos en suelo rural, (ver Figura 5).
Por último, el análisis de la variable pobreza realizado en correlación con cifras de población y situación financiera del municipio, permitió establecer que Facatativá durante el periodo 1995-2009 incrementó su población urbana en un 1.5 $\%$, (ver Figura 6). Lo anterior como respuesta a la diferencia entre nacimientos y defunciones y al cambio de residencia del $51 \%$ de las personas involucradas en dicho crecimiento; este último dado por razones familiares en un $54 \%$, por otras razones el $26 \%$, por dificultad para conseguir trabajo el $12 \%$, y por amenaza para su vida el $2 \%$ (DANE, 2005).

Tabla 1. Barrios de origen informal

\begin{tabular}{|l|r|l|r|r|c|}
\hline $\begin{array}{c}\text { Asentamientos Humanos } \\
\text { de Origen Informal }\end{array}$ & Año & Ubicación & Área/M2 & $\begin{array}{c}\text { \% Area del } \\
\text { total urbano }\end{array}$ & Fecha Formalización \\
\hline${ }^{*}$ Berlin & 1965 & Periferia Norte & 20,808 & 0.3 & 1993 \\
\hline Santa Isabel & 1965 & Periferia Norte & 57,212 & 1.0 & 1993 \\
\hline Los Molinos - Maria Paula & 1965 & Periferia Sur Occidente & 70,737 & 1.2 & 1993 \\
\hline${ }^{*}$ Cartagenita & 1979 & Periferia Sur Oriente & 106,370 & 1.8 & 1993 \\
\hline El Triangulo & 1979 & Periferia Sur Oriente & 12,444 & 0.2 & No se encontró proceso \\
\hline${ }^{*}$ Dos Caminos & 1979 & Periferia Nor Oriente & 14,317 & 0.2 & En proceso \\
\hline${ }^{*}$ La Paz & 1979 & Periferia Sur Central & 40,776 & 0.7 & No se encontró proceso \\
\hline San Cristobal & 1979 & Periferia Sur Occidente & 25,215 & 0.4 & No se encontró proceso \\
\hline${ }^{\star}$ El Jardín & 1988 & Periferia Sur Oriente & 37,350 & 0.6 & En proceso \\
\hline El Paraiso & 1995 & Periferia Sur Central & 16,697 & 0.3 & No se encontró proceso \\
\hline Girardot & 1995 & Periferia Sur Central & 27,132 & 0.5 & No se encontró proceso \\
\hline Nueva Holanda & 1995 & Periferia Nor Oriente & 18,777 & 0.3 & No se encontró proceso \\
\hline${ }^{*}$ Manablanca & 2000 & Periferia Nor oriental & 19,283 & 0.3 & En proceso \\
\hline${ }^{*}$ Chico & 2000 & Periferia Nor oriental & 21,965 & 0.4 & En proceso \\
\hline${ }^{*}$ El Cóndor & 2000 & Periferia Nor oriental & 9,682 & 0.2 & En proceso \\
\hline El Mirador & 2000 & Periferia Sur Central & 12,234 & 0.2 & No se encontró proceso \\
\hline Juan XXII & 2000 & Periferia Sur Occidente & 35,850 & 0.6 & No se encontró proceso \\
\hline TOTAL & & & 546,847 & 9.1 & \\
\hline
\end{tabular}

Fuente: Información POT (Alcaldía Facatativá, 2001). Cálculos y estimaciones propios.

Figura 5. Vista multitemporal proceso de urbanización barrio San Antonio - sector rural, y sectores de Manablanca, actualmente zona urbana de Facatativá.
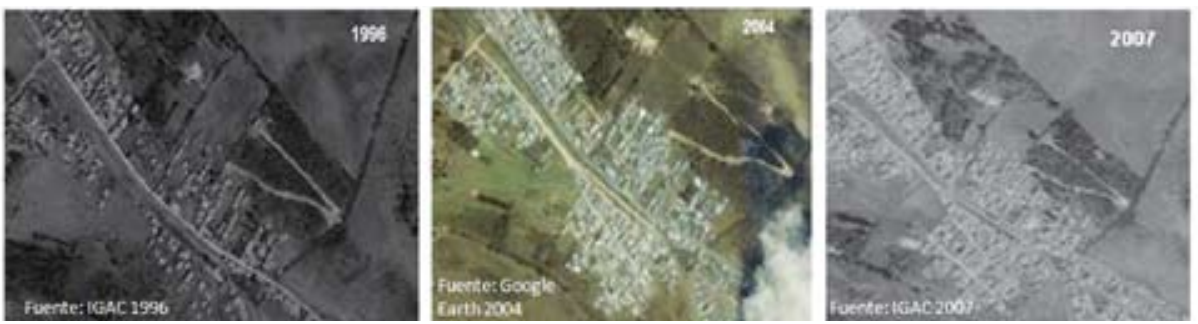
Figura 6. Incremento poblacional acumulado municipal.

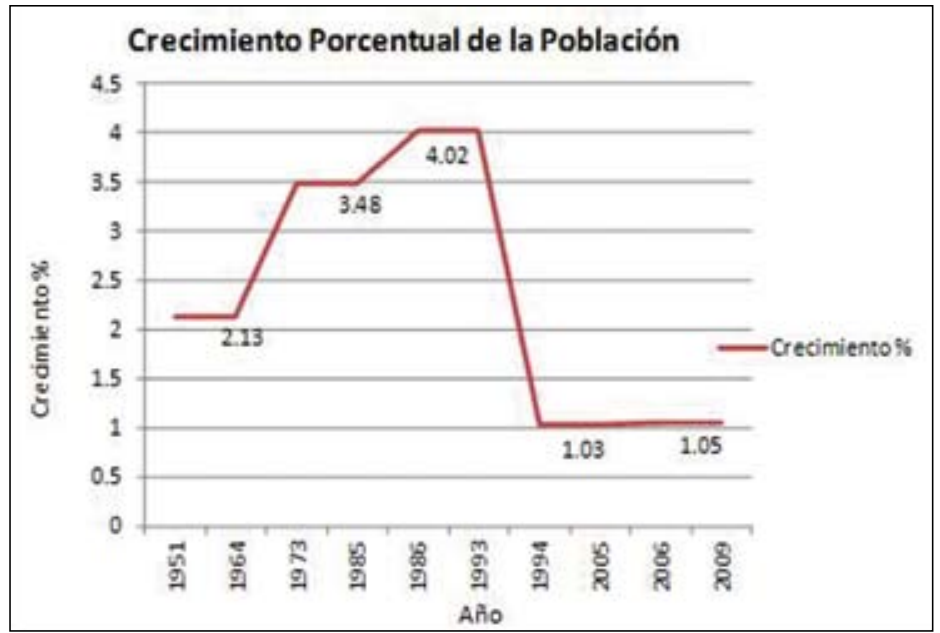

Fuente: DANE (2005). Elaboración propia.

El cambio de residencia en cuestión obedece a la atracción que generó el municipio por sus condiciones económicas y por la demanda de mano de obra no calificada presente en la zona, especialmente por el sector floricultor. Esta dinámica consiguió alcanzar una reducción del 9 $\%$ en las cifras de pobreza durante los años 1995 a 2006, con un decrecimiento final del $1 \%$ para el año 2009, (ver Tabla 3).
Con fundamento en lo expuesto, se concluye que el número de hogares que presentan carencias no aumentó, a pesar del incremento poblacional presente en el municipio, y que la dinámica económica que caracterizó al municipio durante el periodo permitió mejorar la calidad de vida de las familias en términos de características de sus viviendas, dependencia económica y educación para los infantes (indicadores NBI).

Tabla 2. Cifras de pobreza en Facatativá-Cabecera. Periodo 1995-2009

\begin{tabular}{|c|c|c|c|c|c|c|c|c|c|c|c|c|c|c|c|}
\hline \multicolumn{16}{|c|}{ FACTOR POBREZA: Método necesidades básicas insatisfechas NBI } \\
\hline Año & $\stackrel{\text { 용 }}{\stackrel{2}{\circ}}$ & $\stackrel{\circ}{\circ}$ & 용 & $\stackrel{\circ}{\stackrel{\circ}{\circ}}$ & ஓ & ঃ & চ্ণ & స్ రి & ஜ & ষ্ণ & ஜ̊ํํ & ๕ & ¿̊̀ & $\stackrel{\infty}{\circ}$ & ঃ) \\
\hline $\begin{array}{c}\text { Hogares } \\
\text { pobres } \\
(\%)\end{array}$ & 22 & 22 & 22 & 22 & 22 & 22 & 22 & 22 & 22 & 22 & 22 & 13 & 13 & 13 & 12 \\
\hline
\end{tabular}

Fuente: DANE, 2005. Elaboración Propia. 


\section{Conclusiones}

Como resultado de la investigación, se establece que la localización de las viviendas de origen informal en las áreas periféricas de Facatativá responde a los menores precios de la tierra en dichas zonas, ocasionados por la desventaja que representa la distancia que existe entre ellas y el área de servicios de la ciudad, la cual se encuentra en el centro de esta urbe, y que se traduce en aumento en los costos de desplazamientos y en el tiempo requerido para acudir a dicho centro a utilizar los servicios bancarios, médicos, hospitalarios, educativos, etc. Otro aspecto que ocasiona menores precios de la tierra en la periferia lo constituye la dotación de servicios públicos domiciliarios, ya que en algunos de estos sectores periféricos, por las condiciones topográficas, o por las implicaciones económicas que conlleva extender las redes de servicios fuera del perímetro de servicios, las empresas prestadoras no los instalan, por lo tanto las familias deben proveerse de los mismos por cuenta propia.

Con relación a la referida desventaja de ubicación en la periferia, es importante anotar que, no obstante lo citado, dicha desventaja se constituye en ventaja para las familias que habitan los denominados barrios de origen informal en Facatativá, ya que estas acuden solo ocasionalmente al centro de la ciudad, y, por el contrario, se desplazan la mayor parte de la semana hacia las zonas floricultoras de los municipios vecinos, por encontrar allí su fuente de trabajo. De esta manera, la distancia al centro de la ciudad en realidad no es un inconveniente, pues el medio de transporte que utilizan los habitantes de los mencionados barrios para llegar a los cultivos circula por las vías periféricas de la ciudad, es decir, cerca de sus viviendas.

Continuando con los resultados observados, se tiene que en la ciudad de Facatativá el 12,06 \% de los hogares se encuentran en NBI, lo cual significa que dicha población presenta carencias en alguna necesidad básica. De manera que, si se tiene en cuenta que la vivienda es considerada una necesidad básica, se establece que estos hogares, buscando satisfacer dicha carencia, acuden al mercado inmobiliario, el cual, de acuerdo con la lógica del Estado, propiciará el mayor bienestar.

El citado mercado, de acuerdo con las cifras estudiadas, indica que las familias encontrarán ofertas de lotes con diferencias en precios del orden del 88.88 $\%$, fundamentadas en las características citadas en los párrafos anteriores. El referido comportamiento de los precios de la tierra, resultante de la actuación de la lógica de mercado, presenta dos alternativas a las familias con NBI que desean proveerse de vivienda: adquirir el lote en zonas de origen formal mediante un crédito hipotecario o hacerlo en zonas de origen informal, bajo sistemas de financiación no convencionales.

En la práctica, no es posible acudir a la alternativa del sistema financiero mediante crédito hipotecario, pues las carencias de estas familias les impiden cumplir con los requisitos establecidos según la lógica del Estado, por lo tanto, 
actuando bajo la lógica de la necesidad, recurren a la otra alternativa, la oferta informal, conformada por lotes en los sectores rurales periféricos de la ciudad, que se desarrollan de manera irregular, y que, además de presentar precios más bajos, son flexibles en los requisitos para acceder a la financiación.

En el proceso de toma de decisiones por parte de las familias carentes de vivienda, que actúan bajo la lógica de la necesidad, la compra de un lote de la oferta informal parece ser la opción ideal. Así las cosas, la probabilidad de resolver el problema de la vivienda es mayor, pues se ha encontrado precio y financiación accesible, pero prevalecen los inconvenientes de instalarse en una zona de desarrollo informal, puesto que, como se anotaba, estos sectores se caracterizan por presentar carencias en la prestación de servicios públicos domiciliarios y de transporte, y por padecer de problemas de titulación. A este respecto y considerando que la motivación inicial de las familias es conseguir un techo, los referidos inconvenientes pasan a un segundo plano, por lo tanto, la prioridad es comprar el lote para dar inicio a la construcción de la vivienda.

En la fase de construcción de la vivienda, se identifica la necesidad de recuperar la inversión realizada y de garantizar posteriores ingresos derivados de la misma, por lo tanto, en estos sectores, la vivienda se diseña para albergar no solamente a la familia sino a eventuales arrendatarios, como también para instalar en ella un local o un taller. Bajo este contexto, las familias encuentran que las situaciones que antes se presentaban como problemáticas ahora resultan ventajosas.

Una vez constituidos estos barrios de origen informal, hace presencia la administración municipal, bien sea de oficio o a petición de los habitantes, a fin de formalizar el barrio, mediante programas de formalización, adelantando las adecuaciones urbanísticas requeridas, saneando los títulos e incorporando estos predios a las bases de datos con fines fiscales. Sin embargo, cabe anotar que dentro de la investigación no se evidenció que la administración adelantara proceso alguno contra los propietarios de las fincas que fueron parceladas para posteriormente vender lotes sin contar con la respectiva licencia urbanística.

En este orden de ideas, el proceso de producción de vivienda informal, conformado por los elementos pobreza, precio de la tierra y programas de formalización, se constituye en un circuito que se retroalimenta entre sí, con la participación activa de los propietarios de la tierra, de las familias carentes de vivienda y de la administración municipal; lo cual comprueba la hipótesis de que los citados elementos (precio de la tierra, pobreza y programas de formalización), para el caso de Facatativá, si promueven la permanencia del fenómeno.

Como complemento a la situación anteriormente descrita, se observó que la administración municipal, amparándose en su POT como instrumento de planificación, tomó acciones frente al fenómeno 
de la informalidad, articulando el espíritu de la citada Ley Marco de Vivienda con la implementación del programa de vivienda de interés social y vivienda social básica, aprobado en el referido POT. Dicho documento cita que los programas de vivienda social se dirigen a "eliminar la ilegalidad en el proceso de urbanización, a partir de la oferta de proyectos con condiciones económicas acordes a la capacidad de la población" (Alcaldía Facatativá, 2001), favoreciendo a familias con ingresos inferiores a tres salarios mínimos mensuales vigentes (smmlv).

La estrategia empleada por el municipio en el citado programa de vivienda consistió en destinar con anticipación los terrenos para la construcción de vivienda básica social, menor a $60 \mathrm{smmlv}$, ejercer directamente el liderazgo del proyecto, incorporar suelo rural como suelo urbano de expansión y procurar recursos con organizaciones no gubernamentales.

Como resultado de esta gestión, se generaron los proyectos urbanísticos Juan Pablo II y Portal de María, encaminados a cobijar a una parte de las familias (2.848), que son rechazadas por el sector financiero (Alcaldía de Facatativá, 2009).

De esta manera, entendiendo la financiación de la vivienda como la piedra angular del problema, se considera que la experiencia de construir vivienda mediante el descrito sistema participativo implementado en Facatativá, en el que el municipio asigna el subsidio en especie -el suelo-, las familias reúnen la cuota inicial mediante ahorro programado, $y$ las cajas de compensación y el Fondo Nacional de Vivienda FONVIVIENDA otorgan los subsidios para compra de manera anticipada de las viviendas, es una manera adecuada y acertada de enfrentar el problema de la informalidad en la tenencia de la tierra para producir vivienda urbana.

Queda pues en manos de los planificadores colombianos optar por encaminar el esfuerzo y los recursos de las administraciones públicas hacia la prevención de las causas de esta problemática. 


\section{Literatura citada}

Abramo, P. (2003). La teoría económica de la favela: cuatro notas sobre la localización residencial de los pobres y el mercado inmobiliario informal. Río de Janeiro, Brasil: IPPUR-UFRJ.

Alcaldía Municipal de Facatativá. (2002). Decreto 069 de 2002. Por medio del cual se adopta el Plan de Ordenamiento Territorial POT de Facatativá, Colombia.

Departamento Administrativo de la Presidencia de la República (1995). Decreto 755 de 1995. Bogotá, Colombia.

Departamento Administrativo Nacional de Estadística DANE. (2005). Censos de población. Bogotá, Colombia.

Duhau, E. (2002). Dimensiones socio-políticas de la irregularidad y la regularización de los asentamientos populares. México: Azcapotzalco.

Fernandes, E. \& Smolka, M. (2004). Regularización de la tierra y programas de mejoramiento: nuevas consideraciones. Brasil: Recife.

Instituto Geográfico Agustín Codazzi IGAC. (1997). Manual de reconocimiento predial. Bogotá, Colombia: IGAC.

Instituto Geográfico Agustín Codazzi IGAC. (1996). Diccionario Geográfico de Colombia. Tomo 2. Bogotá, Colombia: IGAC.

Instituto Geográfico Agustín Codazzi IGAC. (1985). Metodología para la definición y avalúo de zonas homogéneas (área urbana). Bogotá, Colombia: IGAC.

Recepción: 12 de agosto de 2011

Evaluación: 16 de agosto de 2011

Aprobación: 02 de septiembre de 2011 